\title{
Modeling Prior Shape and Appearance Knowledge in Watershed Segmentation
}

\author{
Xiaoxing Li and Ghassan Hamarneh \\ School of Computing Science \\ Simon Fraser University, Burnaby, BC, V5A 1S6 Canada \\ E-mail: $\{$ xli1, hamarneh $\} @$ cs.sfu.ca
}

\begin{abstract}
Watershed transform is widely used in image segmentation. However, its shortcomings such as over-segmentation and sensitivity to noise often make it unsuitable as an automatic tool for segmenting medical images. Utilizing prior shape knowledge has been demonstrated to improve robustness of medical image segmentation algorithms. In this paper, we propose a novel method for incorporating prior shape and appearance knowledge into watershed segmentation. Our method is based on iteratively aligning a shape-histogram with the result of an improved $k$-means clustering algorithm. No human interaction is needed in the whole process. We demonstrate the robustness of our method through segmenting the corpora callosa from a set of 51 brain magnetic resonance (MR) images. Numerical validation of the results is provided.
\end{abstract}

\section{Introduction}

The concept of watersheds is well known in topography. It was considered as a potential method for image segmentation in [1]. In [2] parallel watershed segmentation was developed as a practical segmentation tool which generated complete contours offering a clear partition of the image. Watershed has been recognized as a popular segmentation algorithm with its simplicity, speed and complete division of the image. However, drawbacks such as over-segmentation and sensitivity to noise prevent watershed transform from being used extensively especially for medical imaging data. Typically, watershed transform is computed on the gradient magnitude of the original image. Over segmentation typically occurs when fluctuation in the gradient image lead to additional catchment basins and subsequently a large number of undesired segments. Furthermore, each negative impulse noise can be considered as a local minimum resulting in an additional labelled segment. In order to overcome these drawbacks several methods have been proposed. Among the most notable is the use of region markers $[3,9,10]$. With region markers, certain desired local minima are selected as markers then geodesic reconstruction is applied to fill all the others to non-minimum plateaus. In this way, the image is segmented into a desired number of segments. However, automatic marker selection is a tedious process requiring human interaction. In addition, geodesic reconstruction can cause important image features to be overlooked if markers are selected inappropriately, changing the final result dramatically. In medical image analysis, an active area of research involves the modeling and analysis of normal and pathological structural shape variability. It has been demonstrated in several medical image segmentation techniques that improved convergence and robustness can be obtained when prior shape knowledge is utilized $[4,5,6,7,8]$.

In this paper we propose a novel method for incorporating prior shape and appearance knowledge into the watershed segmentation technique. The method is composed of a training stage and a segmentation stage. In the training stage, a shape histogram and intensity statistics are used to model prior knowledge. The segmentation stage is a fully automatic iterative procedure and relies on four main steps: classical watershed transform, improved k-means clustering, shape aligning, and refinement.

The rest of the paper is organized as follows. Section 2 discusses the details of the algorithm including modelling prior knowledge (2.1), watershed segmentation (2.2), improved k-means clustering (2.3), current estimate alignment (2.4) and refinement (2.5). In section 3, we apply our method to segmenting the corpora callosa from a set of 51 brain MR images and provide numerical validation of the results. Concluding remarks are given in section 4 .

\section{Methods}

\subsection{Modeling shape and appearance knowledge}

A training set of gray level images containing the object of interest is collected. Additionally, binary images of expert-segmented results identifying the shape of the ob- 
ject of interest are also provided. Fig.1.a shows a subset of expert-segmented corpora callosa from mid-sagittal MRI slices. The binary shape images are aligned by applying a translation, a rotation, and a scaling, in order to normalize the location of the centroid, the direction of the principle axis, and the area of each of the binary shapes, respectively.

A suitable shape model should represent the detailed shape information and be able to model possible shape variations. To this end we provide a modification to the idea of a probabilistic atlas by incorporating additional information derived from the distance transform. Firstly, a shape histogram is obtained by adding the values of corresponding pixels of the set of aligned binary shape images. The value at pixel $p$ in the shape histogram, denoted by $\mathrm{SH}(p)$, reflects the number of shapes containing $p$. The shape histogram of a set of aligned corpora callosa is shown in Fig.1.b.

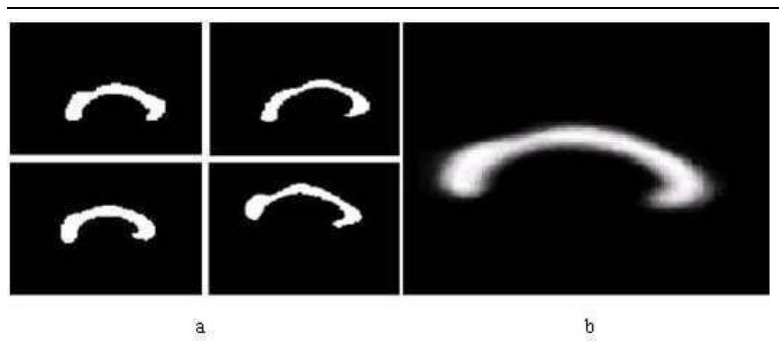

Figure 1. (a) A subset of the training set of corpora callosa binary shape images extracted from mid-sagittal MRI brain slices. (b) The shape histogram of aligned binary images.

Secondly, by overlapping and aligning the shape histogram with the instance of the target structure in a new test image, we can construct a probability map in which the value of each pixel represents the probability that the underlying pixel $p$ belongs to the target. Alignment details are described in section 2.4 . The probability map is denoted by $\operatorname{PM}(p)$ and defined as

$$
\operatorname{PM}(p)= \begin{cases}s_{2}+s_{1} \cdot \operatorname{SH}(p) & \text { if } p \in \Re ; \\ s_{2} \cdot[1-\operatorname{Dist}(p)] & \text { if } p \notin \Re .\end{cases}
$$

where $\Re$ is the set of nonzero pixels in the shape histogram. $\operatorname{Dist}(p)$ is the normalized two-dimensional Euclidean distance transform for the binary image having $\Re$ as foreground [13]. The two scalars, $s_{1}$ and $s_{2}$, satisfy $s_{1}+s_{2}=1$ and determine the weight of shape information when constructing the probability map. An example of the probability map is shown in Fig.2.a and Fig.2.b plots $\operatorname{PM}(p)$ of the pixels along the straight line across the probability map in Fig.2.a.

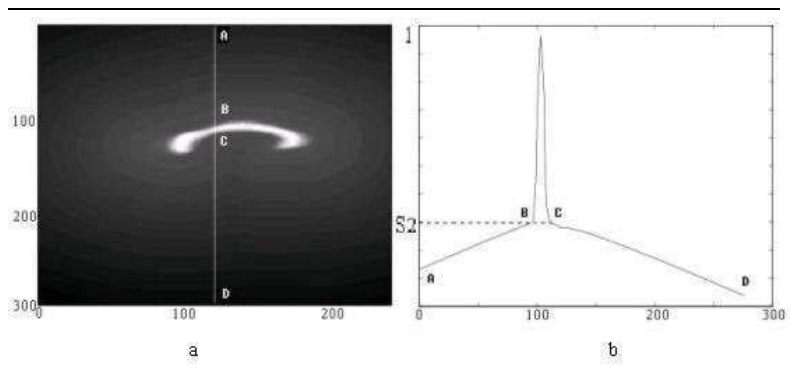

Figure 2. (a) Probability map. (b) Probability profile along the line ABCD in (a).

In addition to capturing shape variation information from the expert-segmented binary images, we model the appearance knowledge from the intensity patch of the object of interest in the training set of gray level images. In medical images, anatomical structures have characteristic intensity features that can be utilized when locating similar structures in new images. Histogram equalization is first applied to the training set of gray level images and then the mean, $M_{a p p}$, and variance, $V_{a p p}$, of the intensity patches are calculated and used as two appearance descriptors.

\subsection{Watershed transform}

By utilizing prior knowledge our method will be able to cope with the over segmentation problems of the standard watersheds transform. In this section we present a review of the standard watershed segmentation algorithm and follow the notations of [11] for the discrete case using topographical distance. The reader is referred to [11] for a detailed treatment.

The lower slope, $\operatorname{LS}(p)$, is the maximum slope connecting pixel $p$ in the input image $f$, with its neighbors of lower altitude, which can be written as

$$
\operatorname{LS}(p)=\max _{q \in N(p) \cup p}\left(\frac{f(p)-f(q)}{d(p, q)}\right)
$$

$N(p)$ is the set of neighbors of pixel $p$ and $d(p, q)$ is the Euclidean distance between $p$ and $q$. Note that when $q=p$, the right hand side of (2) is forced to be zero; thus we have a lower slope value even when $p$ is a local minimum. Consequently, the cost for moving from pixel $p$ to $q$ is defined 
as

$$
\operatorname{cost}(p, q)= \begin{cases}\operatorname{LS}(p) \cdot d(p, q) & \text { if } f(p)>f(q) \\ \operatorname{LS}(q) \cdot d(p, q) & \text { if } f(p)<f(q) \\ \frac{1}{2}[\operatorname{LS}(p)+\operatorname{LS}(q)] \cdot d(p, q) & \text { if } f(p)=f(q) .\end{cases}
$$

If there exists a path $\pi=\left(p_{0}, \cdots, p_{l}\right)$ from $p_{0}=p$ to $p_{l}=q$, the topographical distance along $\pi$ between the two pixels $p$ and $q$ is expressed as

$$
T_{f}^{\pi}(p, q)=\sum_{i=0}^{l-1} d\left(p_{i}, p_{i+1}\right) \operatorname{cost}\left(p_{i}, p_{i+1}\right)
$$

$T_{f}^{\pi}(p, q)$ is the minimum of the topographical distances of all paths linking $p$ and $q$. Following similar definition, the catchment basin $\mathrm{CB}\left(m_{i}\right)$ of a local minimum $m_{i}$ is defined as the set of pixels which have smaller topographical distances to $m_{i}$ than any other local minimum. Finally, the set of pixels which do not belong to any catchment basin are referred to as the watershed pixels. Note that, in practice, the watershed transform is usually computed on the gradient magnitude image instead of the gray value image. An example of watershed transform is shown in Fig.3.c

\subsection{Improved $k$-means clustering}

After performing a standard watershed transform, we make use of the k-means clustering algorithm to assign the resulting segments into different classes. K-means is a clustering algorithm that assigns $N$ data points to $k$ disjoint subsets, $S_{j}, j=1,2, \cdots, k$, each containing $N_{j}$ data points, by minimizing the sum-of-squares criterion given by

$$
J=\sum_{j=1}^{k} \sum_{n \in S_{j}} \sqrt{\left|x_{n}-\mu_{j}\right|^{2}}
$$

where $x_{n}$ is the value of $n$th data point and $\mu_{j}$ is the mean value of the data points within the cluster $S_{j}$.

K-means clustering algorithm can effectively assign the watershed segments into several clusters according to different features. In our algorithm, we jointly utilize two kinds of features for each segment; (i) the mean normalized intensity and (ii) the spatial centroid position of each segment. For the $n$th segment, these features are denoted by $I_{n}$ and $C_{n}$, respectively. Accordingly, we design an improved kmeans clustering scheme with a modified sum-of-squares criterion, given by

$$
J^{\prime}=\sum_{j=1}^{k} \sum_{n \in S_{j}} \sqrt{\left|I_{n}-\eta_{j}\right|^{2}+\alpha\left|C_{n}-\mu_{j}\right|^{2}}
$$

where $\eta_{j}$ and $\mu_{j}$ denote the average values of $I_{n}$ and $C_{n}$, respectively, $\forall n$ belonging to the $j$ th cluster. By minimizing (6), each resulting cluster of watershed segments will contain segments with similar intensity. Furthermore, the watershed segments within each cluster tend to be in close proximity and the likelihood of assigning non-neighboring segments to the same cluster is reduced. The parameter $\alpha$ determines the weight for penalizing distant segments when clustering. The value of $\alpha$ is chosen to be inversely proportional to the average distance between the two farthest points of an object in the training set (zooming). An excessively large $\alpha$, for example, can hinder segments belonging to a long, thin structure from being grouped together.

To overcome the sensitivity of the k-means algorithm to different initializations, we order the segments according to their mean intensities and assign the same number of segments to each of the $k$ clusters.

After clustering, we locate an initial estimate of the target structure by selecting the cluster whose intensity profile is most similar to the appearance knowledge obtained from the training set (section 2.1). Specifically, after histogram equalization, we calculate the mean and variance of the intensity for each cluster. We then choose the cluster having the most similar intensity statistics to those calculated in the training. This is expressed as finding the $j$ th cluster that minimizes

$$
\mathrm{DF}(j)=\sqrt{\left(\mathrm{m}_{j}-M_{a p p}\right)^{2}+\left(\mathrm{v}_{j}-V_{a p p}\right)}
$$

where $\mathrm{m}_{j}$ and $\mathrm{v}_{j}$ denote the mean and variance of the intensity of the $j$ th cluster, respectively. The set of all watershed pixels belonging to this selected cluster is considered to be

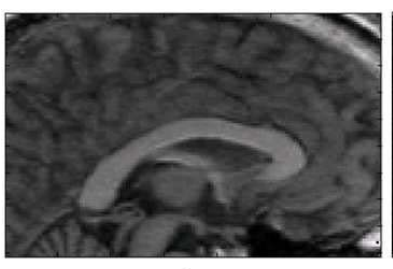

a
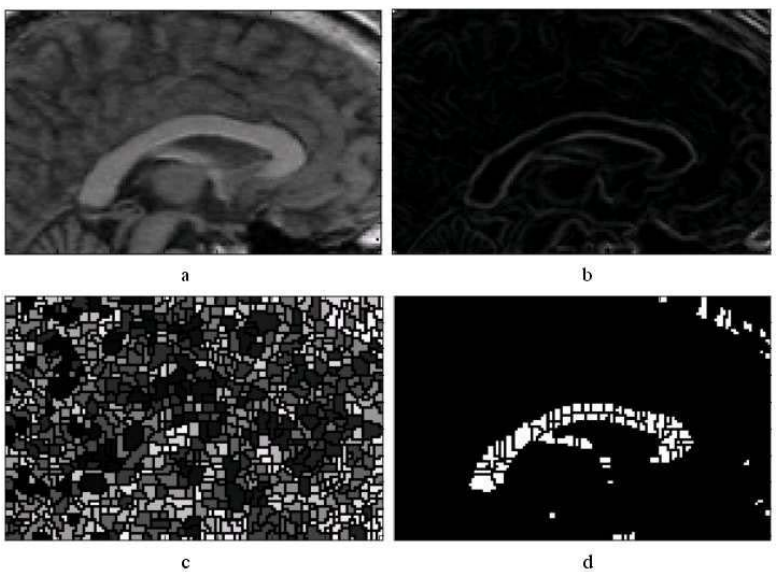

Figure 3. Calculating the initial estimate of the corpora callosa segmentation. (a) Original mid-sagittal MR image. (b) Gradient magnitude image. (c) Watershed transform. (d) Result of improved k-means clustering and initial segmentation estimate. 
the initial segmentation estimate and will be iteratively improved (section 2.4). Fig. 3 demonstrates initializing the first estimate of the corpora callosa segmentation by applying our improved k-means clustering to a watershed segmentation of an MR image. Note how the watershed segmentation is characterized by excessive over-segmentation (Fig.3.c).

Note also how the clustering algorithm detects a good approximation of the corpora callosa (Fig.3.d) with some undesired segments (upper right corner of Fig.3.d).

\subsection{Iterative shape alignment}

In this section we iteratively merge segments and eliminate undesired ones by aligning the current estimate with the shape histogram. Initially however, we perform morphological closing operation on the initial estimate (obtained in section 2.3) to join the segments separated by watershed pixels, the result of which is called target $T$. Now, in the $i$ th iteration of the shape alignment step, the area, orientation and centroid of the current target $T_{i}$ are calculated. Then the shape histogram is scaled, rotated and translated (using bi-cubic interpolation) to be aligned with $T_{i}$, and a probability map is constructed accordingly. Finally the pixels in $T_{i}$ with the lowest probabilities are removed, resulting in an updated target $T_{i+1}$. The $i$ th iteration of this procedure can be expressed by the following steps:

Step 1. Align: Resize, rotate and translate the shape histogram to align it with the target

$$
\mathrm{SH}_{i}=R\left[\left(A_{T_{i}} / A_{\Re}\right) \cdot \mathrm{SH}\right]+\left(C_{T_{i}}-C_{\Re}\right) ;
$$

Step 2. Probability map: Construct the probability map $\mathrm{PM}_{i}$ based on $\mathrm{SH}_{i}$ according to (1);

Step 3. Remove: The pixels in target $T_{i}$ having the lowest probability are removed.

$$
\begin{aligned}
& T_{i+1}=\left\{p \mid p \in T_{i} \cap\right. \\
& \left.\operatorname{PM}_{i}(p)>\sigma \max _{q \in T_{i}}\left(\mathrm{PM}_{i}(q)\right)+(1-\sigma) \min _{q \in T_{i}}\left(\mathrm{PM}_{i}(q)\right)\right\}
\end{aligned}
$$

In the above procedure, $\mathrm{SH}$ represents the shape histogram obtained from the training set of images. $A_{\Re}$ and $C_{\Re}$ are the area and centroid of the nonzero region $\Re$ of the shape histogram, respectively. Similarly, $A_{T_{i}}$ and $C_{T_{i}}$ are the area and centroid of the target $T_{i}$, respectively. By multiplying the rotation matrix $R$, the shape histogram will rotate by a degree of $\Theta=\theta_{T_{i}}-\theta_{\Re}$. $\left(\theta_{T_{i}}\right.$ and $\theta_{\Re}$ are the orientations of the principle axes of $T_{i}$ and $\Re$, respectively). $\sigma$ is the step size associated with pixel removal for each iteration. A small $\sigma$ generates a more accurate result but requires more iterations for the algorithm to converge.

The algorithm converges when all the remaining pixels have probabilities larger than $s_{2}$, which indicates that the

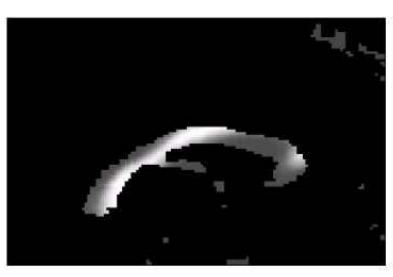

a

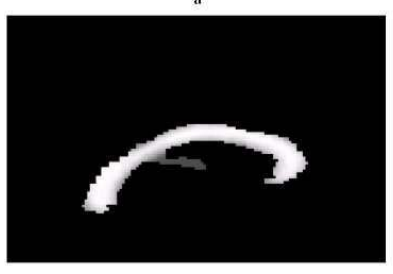

c

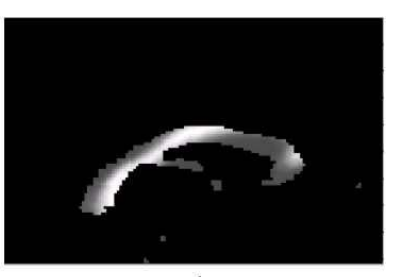

b

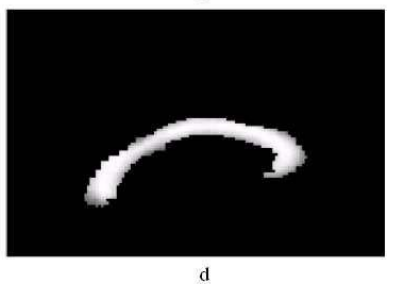

Figure 4. Iterative shape alignment. (a) Initial alignment, (b) 1st iteration, (c) 3rd iteration, and (d) the algorithm converges after the 5th iteration. The gray intensity values represent the values of the probability map.
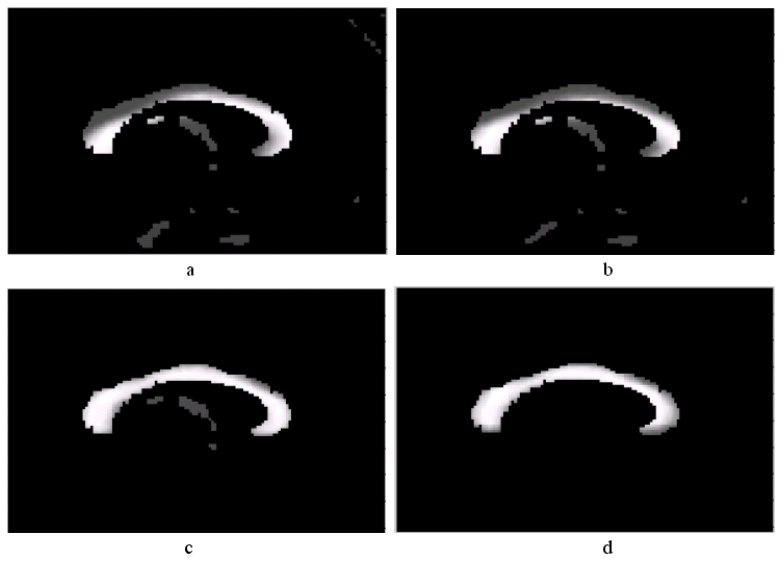

Figure 5. Iterative shape alignment. (a) Initial alignment, (b) 1st iteration, (c) 2nd iteration, and (d) the algorithm converges after the 4th iteration.

shape of the current target is similar enough to the shape histogram and the desired structure is well segmented. Two sets of intermediate results are shown in Fig.4 and Fig.5. We can see some difference between the two initializations, however both cases generated good alignments. The two cases converge after five and four iterations, respectively. Using the target obtained in the final iteration, we can determine which segments should be kept. These segments are merged together to form the segmented result of the desired organ. 

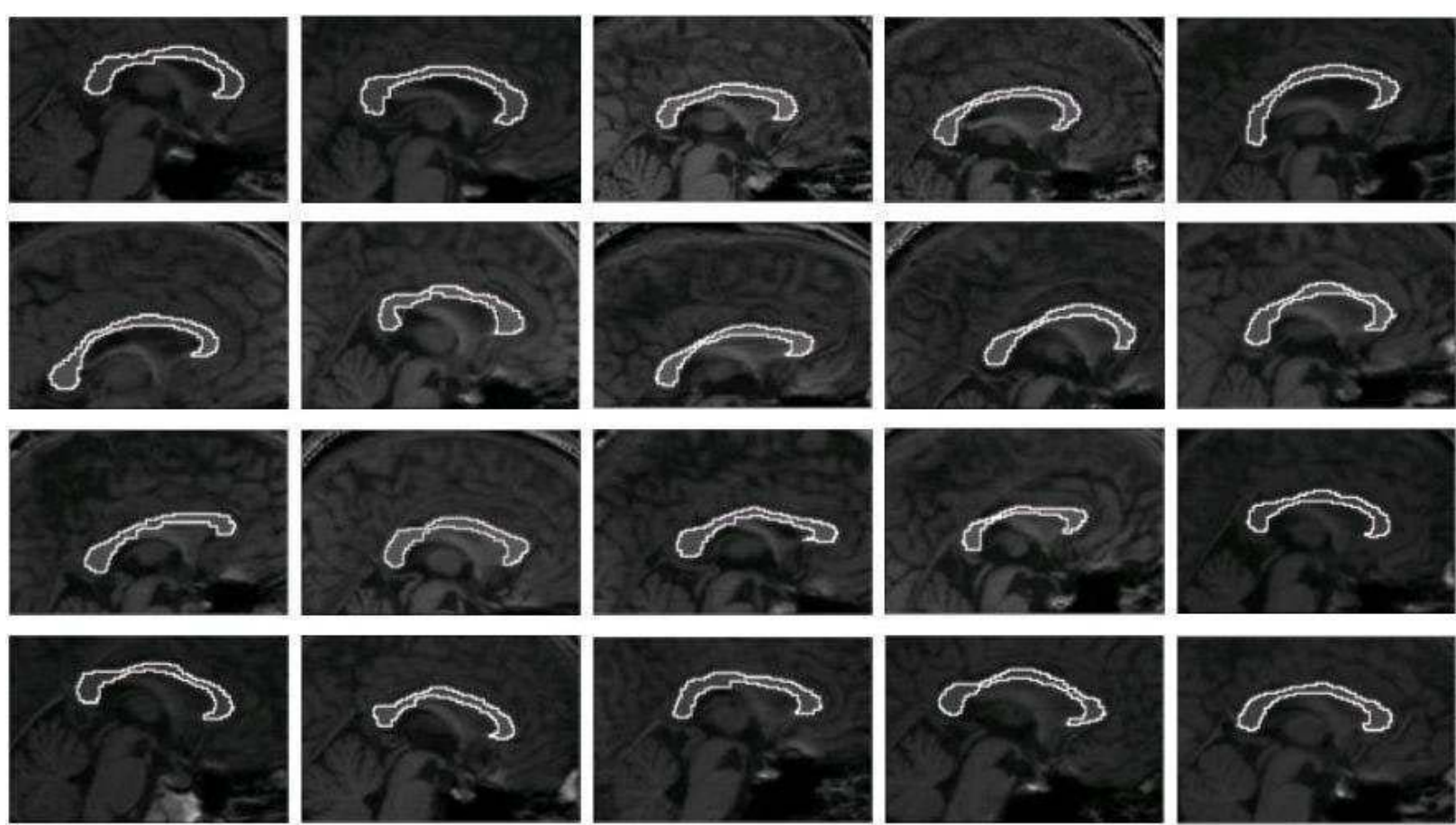

Figure 6. Some example segmentation results.

\subsection{Refining segmentation results}

If the input image data is noisy, the edge of the final segmented result acquired in section 2.4 may be not smooth. In fact, some small marginal segments may get lost during the clustering step since their mean intensities are not similar enough to the segments inside the organ. Hence, it was found useful to merge some of the one ring segments if they are covered by $\Re$ and have the similar mean intensities as that of the target structure.

\section{Results}

Our proposed algorithm has been tested on 51 brain MR images [12]. Expert-segmented corpora callosa for all images in the data set were provided. To ensure cross validation, when segmenting an image, the corresponding expertsegmented result and appearance information were not included in the training set, i.e. a leave-on-out scheme was applied in which 50 MR images with the corresponding expert segmentation were used as the training set for segmenting the remaining one image. The shape histogram is obtained by aligning and adding the expert segmented results. Each test image contains a brain region cropped from the original image with a dimension of $91 \times 141$. The cropped brain parts of the 51 MR images mostly cover the region begin- ning at the calvaria, reach the upper part of the pons and have a fixed aspect ratio. The intensity and contrast of these images vary from one to another and the shape variation of corpora callosa is considerably large. The original image has been preprocessed by a median filter prior to computing the gradient.

A constant value of $\alpha=0.5$ for all the MR images was used. By selecting $\alpha<1$, we actually put more emphasis on intensity information and use spatial position as additional constraints when clustering the segments. We choose $\sigma=2.5 \%$ as the removal step size in each iteration. In general, for the less noisy images, $\sigma$ can be larger since there will not be many small segments spreading around the structure. However, to ensure the accuracy of the results, it should be no larger than $5 \%$. On the other hand, for the images with much more noise, $\sigma$ should be smaller and the algorithm will require more iterations to get converged. In our experiment, most cases converged in less than 5 iterations. The two scalars $s_{1}$ and $s_{2}$ in (1) are selected to be $s_{1}=0.7$ and $s_{2}=0.3$. We always use $s_{1}>s_{2}$ since shape information is the dominant factor.

All 51 brain MR images were automatically segmented. The results are compared with the corresponding expert segmented ones in the original data set. To demonstrate the accuracy of our method, the maximum, average and standard deviation of Hausdorff distance [14] are calculated and re- 
ported for all the cases (Table 1). It can be seen that our results differ very slightly from the expert segmented results. Fig.6 presents some of the segmented corpora callosa outlined by a white boundary.

However, we report two failure cases in which a satisfactory result can not be generated automatically. One failure case is image No.22. The problem is traced-back to the intrinsic limitation of k-means algorithm, namely the difficulty in determining the optimal number of clusters $k$. Fortunately, in our method, two factors helped in finding a robust $k$ for all the remaining images. The first factor is the similar zooming (as descried in 2.3). The second is that all images of the brain have identical components; white matter, gray matter and CSF. As a result, the intensity distribution of all brain MR images is concentrated around certain intensity ranges. We used an experimental value of $k=7$ and obtained satisfactory results for all the images except image No.22, which had very low contrast. Consequently, k-means algorithm did not successfully classify the segments belonging to the corpora callosa into the same cluster. For that case only, we adjust $k$ and a good result could still be obtained with $k=4$. In Table 1, this case is marked with * and the maximum, average and standard deviation of Hausdorff distance are all calculated using $k=4$.

Another failure case is image No.31. The middle part of the corpora callosa in this image is very thin and noisy as shown in Fig.7.a. The watershed transform was unable to distinguish this part as a separate segment. Consequently, only the two ends of corpora callosa were well segmented and the result is hence broken in the middle, as shown in Fig.7.b.
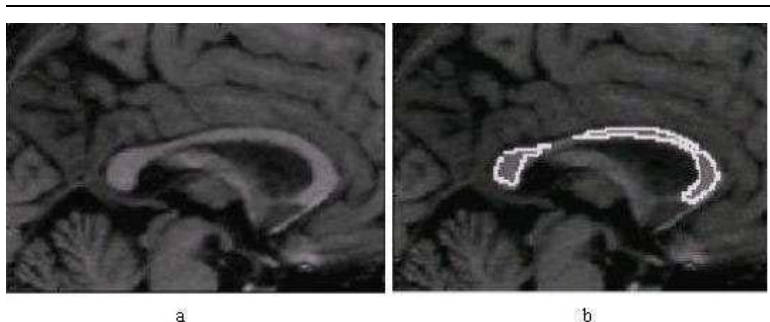

Figure 7. A failure case. (a) Original image No.31. (b) Segmentation result.

\section{Conclusions}

In this paper we propose a new approach to improve watershed segmentation by incorporating shape and appearance knowledge. The method overcomes some intrinsic problems with watershed transform. The over-segmentation

\begin{tabular}{c|ccc}
\hline MRI No. & $\begin{array}{c}\text { Max error } \\
\text { (pixels) }\end{array}$ & $\begin{array}{c}\text { Mean error } \\
\text { (pixels) }\end{array}$ & $\begin{array}{c}\text { SD error } \\
\text { (pixels) }\end{array}$ \\
\hline 1 & 3.6056 & 0.4451 & 0.6340 \\
2 & 4.0000 & 0.6514 & 0.7902 \\
3 & 3.1623 & 0.5835 & 0.5784 \\
4 & 2.0000 & 0.5212 & 0.5737 \\
5 & 2.2361 & 0.4004 & 0.5269 \\
6 & 2.2361 & 0.5205 & 0.5441 \\
7 & 2.0000 & 0.5148 & 0.5427 \\
8 & 2.0000 & 0.5994 & 0.5286 \\
9 & 3.0000 & 0.4962 & 0.5723 \\
10 & 3.6056 & 0.5975 & 0.6183 \\
11 & 2.2361 & 0.3973 & 0.5350 \\
12 & 3.1623 & 0.6404 & 0.6677 \\
13 & 2.8284 & 0.5402 & 0.7000 \\
14 & 2.0000 & 0.4812 & 0.5288 \\
15 & 1.4142 & 0.3367 & 0.4777 \\
16 & 3.6056 & 0.5143 & 0.6551 \\
17 & 4.0000 & 0.4762 & 0.6633 \\
18 & 1.4142 & 0.4034 & 0.4954 \\
19 & 2.0000 & 0.5200 & 0.5258 \\
20 & 1.4142 & 0.3430 & 0.4794 \\
21 & 2.0000 & 0.4075 & 0.5302 \\
$22 *$ & 2.0000 & 0.5234 & 0.5495 \\
23 & 2.8284 & 0.6127 & 0.6517 \\
24 & 2.2361 & 0.4324 & 0.5364 \\
25 & 2.2361 & 0.5491 & 0.5530 \\
26 & 3.0000 & 0.5519 & 0.6029 \\
27 & 3.6056 & 0.5179 & 0.6969 \\
28 & 2.0000 & 0.5019 & 0.5279 \\
29 & 2.0000 & 0.5335 & 0.5694 \\
30 & 3.0000 & 0.5929 & 0.6114 \\
31 & - & - & - \\
32 & 2.0000 & 0.3944 & 0.5164 \\
33 & 2.2361 & 0.4308 & 0.5503 \\
34 & 2.0000 & 0.4907 & 0.5152 \\
35 & 1.4142 & 0.4407 & 0.5118 \\
36 & 3.1623 & 0.5894 & 0.5674 \\
37 & 2.8284 & 0.5002 & 0.6280 \\
38 & 3.6056 & 0.4650 & 0.6325 \\
39 & 1.4142 & 0.4198 & 0.5035 \\
40 & 1.4142 & 0.3758 & 0.4930 \\
41 & 2.0000 & 0.4346 & 0.5231 \\
42 & 2.0000 & 0.4231 & 0.5252 \\
43 & 1.4142 & 0.4716 & 0.5155 \\
44 & 2.0000 & 0.4516 & 0.5485 \\
45 & 2.0000 & 0.5768 & 0.5212 \\
46 & 3.6056 & 0.5109 & 0.6629 \\
47 & 1.0000 & 0.3304 & 0.4704 \\
48 & 2.0000 & 0.3650 & 0.4889 \\
50 & 2.4142 & 0.4785 & 0.5131 \\
\hline & 1.4142 & 0.3875 & 0.5538 \\
\hline & 2.3716 & 0.5364 & 0.5319 \\
\hline & 0.7927 & 0.0806 & 0.5648 \\
\hline & & & 0.0678 \\
\hline
\end{tabular}

Table 1. The mean, maximum, and standard deviation of the Hausdorff distances between our result and expert segmented corpora callosa boundaries. $\left({ }^{*} k=4\right)$

problem is handled by clustering and merging the watershed segments. The effect of noise is suppressed by computing the mean intensity of each segment. As a result, even when the input image has a relatively low resolution and low contrast, our algorithm achieves relatively accurate result. Our method is marginally affected by some of the limitations of the k-means clustering algorithm and a failure case is reported. However, this case can be detected by comparing the segmentation result with the shape histogram and adjusting the number-of-clusters parameter in k-means to ob- 
tain a satisfactory result.

Future work includes applying our method to other data sets as well as extending it to 3D. Given that the main building blocks of our method (watershed, k-means) are well developed in 3D, we foresee no major difficulties. Additionally, more advanced appearance models may be used when the intensity variations within the target structures are more complex as in active appearance models [15]. This was not an issue for the corpora callosa segmentation application.

\section{Acknowledgment}

This research was supported in part by Faculty of Applied Science at Simon Fraser University.

\section{References}

[1] S. Beucher and F. Meyer, "The Morphological approach to segmentation: The watershed transform," Mathematical Morphology in Image Processing, E. R. Dougherty, Ed. New York: Marcel Dekker, vol. 12, pp. 433 - 481, 1993

[2] A. N. Moga and M. Gabbouj, "Parallel image component labeling with watershed transformation," IEEE Trans. Pattern Anal. Machine Intell., vol. 19, pp. 441-450, May 1997

[3] V. Grau, A. U. J. Mewes and M. Alcañiz, "Improved Watershed Transform for Medical Image Segmentation Using Prior Information," IEEE Trans. Medical imaging, vol. 23, No. 4, pp. 447-458, April 2004

[4] T.F. Cootes and C.J. Taylor, "Statistical models of appearance for medical image analysis and computer vision," Proc. SPIE Medical Imaging, 2001

[5] T. McInerney, G. Hamarneh, M. Shenton, D. Terzopoulos, "Deformable organisms for automatic medical image analysis," Medical Image Analysis, vol. 6, pp. 251-266, 2002

[6] M. Leventon, W. Grimson, O. Faugeras, "Statistical Shape Influence in Geodesic Active Contours," Proceed. Computer Vision and Pattern Recognition, pp. 1316-1323, 2000

[7] G. Szekely, A. Kelemen, C. Brechbuehler, G. Gerig, "Segmentation of 3D objects from MRI volume data using constrained elastic deformations of flexible Fourier surface models," Medical Image Analysis, vol. 1(1), pp. 19-34, 1996

[8] G. Hamarneh, T. Gustavsson, "Statistically Constrained Snake Deformations," Proc. Systems, Man, and Cybernetics, vol. 3, pp. 1610-1615, 2000

[9] J. L. Vincent, "Morphological grayscale reconstruction in image analysis: Application and efficient algorithms," IEEE Trans. Image Processing, vol. 2, pp. 176-201, 1993

[10] S. Beucher "Watershed, hierarchical segmentation and waterfall algorithm," Mathematical Morphology and Its Applications to Image Processing, Dordrecht, The Netherlands: Kluwer pp. 69-76, 1994

[11] J. B. T. M. Roerdink and A. Meijster, "The Watershed Transform: Definitions, Algorithms and Parallelization Strategies," Fundamenta Informaticae, vol. 41, pp. 187-228, 2000
[12] M. E. Shenton, R. Kikinis, F. A. Jolesz, S. D. Pollak, M. LeMay, C. G. Wible, H. Hokama, J. Martin, D. Metcalf, M. Coleman, and R. W. McCarley, "Abnormalities in the left temporal lobe and thought disorder in schizophrenia: A quantitative magnetic resonance imaging study," New England Journal of Medicine, vol. 327, pp. 604-612, 1992

[13] H. Breu, J. Gil, D. Kirkpatrick, and M. Werman, "Linear Time Euclidean Distance Transform Algorithms," IEEE Trans. Pattern Anal. Machine Intell., vol. 17, No. 5, pp. 529533, May 1995

[14] P. K. Agarwal and M. Sharir, "Efficient Algorithms for Geometric Optimization," ACM Computing Surveys., vol. 30, No. 4, pp. 412-458, December 1998.

[15] T. F. Cootes, G. J. Edwards, C. J. Tayloe, "Active Appearance Models," IEEE PAMI., vol. 34, No. 6, pp. 681-685, 2001. 